\title{
Nature Metabolism: year one
}

\author{
As we celebrate 1 year at Nature Metabolism, we are grateful for the positive reception from our research \\ community, and we reflect on the past 12 months and on what lies ahead.
}

O ne year ago, we provided readers with a first glimpse at Nature Metabolism and our vision for the journal. Fast forward 12 months, and we are now overwhelmed by the positive response from the scientific community and the captivating content that we have published in our first year. Let's have a closer look.

Our goal is to bring exciting original research articles to the broad community of metabolism researchers. In our first 12 issues, we published 77 primary research articles-in fields as diverse as fundamental cell biology and basic biomedical and translational researchcovering most areas of our scope. One of our most popular topics was metabolic diseases and the physiology of metabolic organs-including adipose tissue, the pancreas and the liver-probably as a reflection of the high quality of research produced in this growing field and the enthusiastic response to the journal from researchers working in the area.

Other research articles in our pages covered a diverse range of subjects including metabolism in cancer cells, immune cells, the vasculature, mitochondrial and bone biology, and studies at the interface of nutrition and organismal ageing. We also published research shedding new light on the regulation of cellular metabolism as well as metabolic research involving model organisms, and we are eager to continue providing a home for such studies in the future. We also dipped our toes into more translational and clinical research, in studies of the genetics of metabolic traits and microbiome research-areas that we would like to see growing further in the journal in the future.

In our commissioned content, we were able to share 15 original Reviews and Perspectives, including three that are part of our ongoing Metabolic Messengers article series. With a total of 40 News \& Views articles, Nature Metabolism connected readers with cutting-edge metabolism research from our pages and a spectrum of journals, and provided detailed analysis and contextualization of the research significance. Finally, with five Comment articles, we provided a forum for lively debate, highlighting key issues and questions in the field while pointing out potential solutions. For example, we have directed

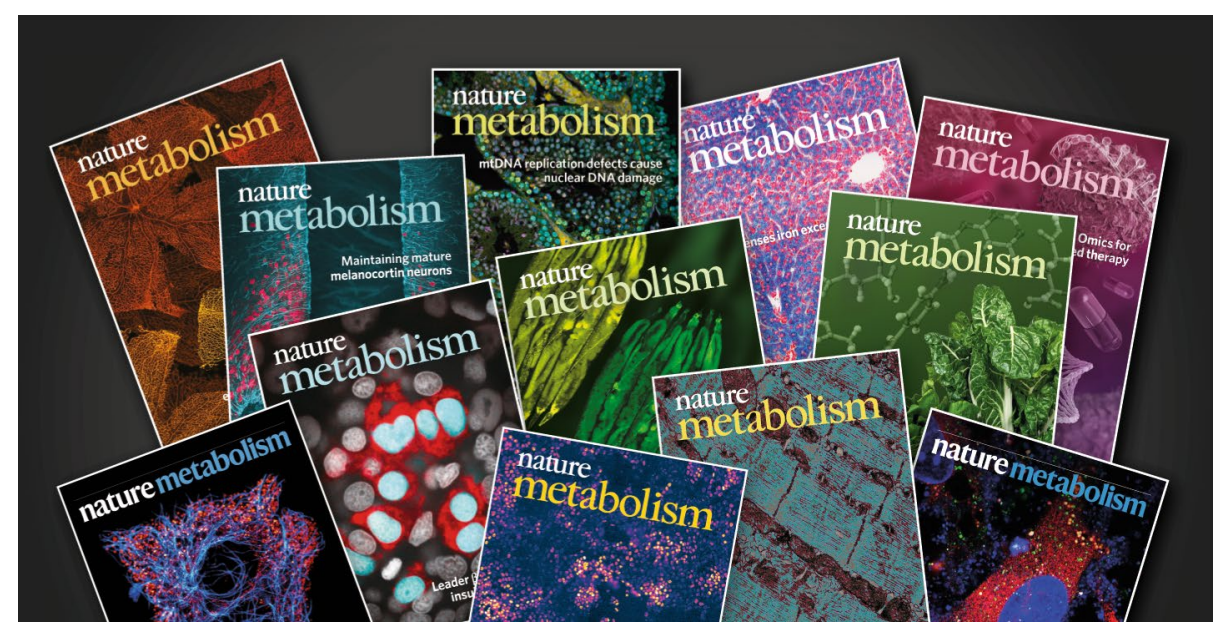

the attention of the metabolism community to key experimental variables, such as the mouse substrains used in animal research and the oxygen concentrations in cell culture experiments, and to calls for increased standardization in lipidomics research. Because we are keen to publish more Comment articles, we welcome suggestions for topics that you would like to see covered in this section.

We have assembled a collection of highlights from our first year, which can be found in our online anniversary collection, available free of charge until the end of the year. We encourage you to have a look.

We would like to take this opportunity to thank all our authors, who have entrusted us with their exciting work during this early phase of the journal. Your support is our motivation behind our ongoing efforts to put together a journal that showcases exciting research and positively affects the wider metabolism community, and that we hope will be essential reading to researchers working in this thriving field.

We were particularly thrilled to receive submissions both from established groups and from young principal investigators, including several studies that were the first papers coming out of the laboratories of newly established groups. We were honoured to share in the excitement of these talented researchers and to provide a platform for these fine studies. We hope that they will receive the attention that they deserve in our pages and that we can serve as a springboard for the careers of the next generation of scientists.

We are also very grateful to all the referees who worked with us to ensure that the research published in Nature Metabolism meets the expectations of the community and the high standards that we are held to by our peers in the field.

Over the past year, we as editors have been humbled by how positively the community has responded to Nature Metabolism. This enthusiasm was particularly palpable during institute visits and scientific conferences, including the two Nature Conferences that we co-organized: Cellular Metabolism in Xiamen, China, and Metabolic Communication in Rio de Janeiro, Brazil. Both events succeeded in bringing the wider metabolism community together in unique ways. Facilitating such interaction and exchange of ideas between researchers working in different subfields of metabolic research is exactly what Nature Metabolism is about.

Turning our attention briefly to our current January issue, we are kicking the year off with a comprehensive review of $\mathrm{NAD}^{+}$metabolism by Auwerx and colleagues. In the primary research section, you can find articles on nutrient sensing and signalling, cancer metabolism, adipose tissue and pancreas biology.

What else is in store for Nature Metabolism in 2020? You can expect us to build on the foundation laid in our first year and to continue to publish exciting 
and thought-provoking research and commentary from the wider metabolism field. We are working to increase the diversity of content that we publish, in terms of not only covering more areas in the journal's scope but also expanding the geographic distribution of our authors. We have some special content planned for later in the year, and we are looking forward to co-organizing another Nature Conference on Metabolism in Health and Disease in April in Wuxi, China. One thing that will remain unchanged is our focus on author service and our commitment to championing the needs of the wider metabolism community.

The first year at Nature Metabolism has been exciting, productive and busy. Getting used to monthly production deadlines posed a challenge for all of us, particularly when travelling. We are grateful for the assistance and encouragement from our colleagues across the business, including those at other Nature journals, who have been hugely helpful in getting us editors through this year and bringing Nature
Metabolism to where it is now. Most importantly, however, we would like to again thank our authors, reviewers and readers for your support. If you have any feedback for us, please do get in touch with us via e-mail or on Twitter (@NatMetabolism).We look forward to working with you on the next 12 issues and beyond.

Published online: 23 January 2020

https://doi.org/10.1038/s42255-020-0168-y 